\title{
Borel exceptional values of meromorphic solutions of Painlevé III difference equations
}

\author{
Jilong Zhang ${ }^{1 *}$ and Hongxun $\mathrm{Yi}^{2}$
}

\author{
"Correspondence: \\ jilongzhang2007@gmail.com \\ ${ }^{1}$ LMIB and School of Mathematics \\ and Systems Science, Beihang \\ University, Beijing, 100191, P.R. China \\ Full list of author information is \\ available at the end of the article
}

\begin{abstract}
In this paper, we investigate Borel exceptional values of meromorphic solutions of Painlevé III difference equations. In particular, let $w$ be a transcendental meromorphic solution of $\bar{w} \underline{w}(w-1)=\eta(w-\lambda)$ with finite order, where $\eta(\neq 0), \lambda(\neq 0,1)$ are constants. If $a, b$ are two Borel exceptional values of $w$, then $a+b=1+\eta$ and $a b=\lambda=\eta^{2}$.
\end{abstract}

MSC: $30 \mathrm{D} 35 ; 39 \mathrm{~A} 10$

Keywords: Borel exceptional values; meromorphic solution; difference

\section{Introduction}

Let $w$ be a meromorphic function in the complex plane. The $z$-dependence is supposed by writing $\bar{w} \equiv w(z+1)$ and $\underline{w} \equiv w(z-1)$. We assume the reader is familiar with the standard notation and results of Nevanlinna value distribution theory (see, e.g., [1-3]). $\rho(w)$, $\lambda(w)$, and $\lambda(1 / w)$ denote the order, the exponents of convergence of zeros and poles of $w$, respectively. We also denote by $S(r, w)$ any quantity satisfying $S(r, w)=o(T(r, w))$ for all $r$ outside of a set with finite logarithmic measure.

Meromorphic solutions of complex difference equations have become a subject of great interest recently [4-9], due to applications of value distribution theory to difference expressions [10-12]. In particular, Halburd and Korhonen [13] studied the difference equation

$$
\bar{w}+\underline{w}=R(z, w),
$$

where $R$ is rational in $w$ and meromorphic in $z$ with slow growth coefficients. They proved that if (1.1) has an admissible meromorphic solution of finite order, then either $w$ satisfies a difference Riccati equation or (1.1) can be transformed into eight simple difference equations. These simple difference equations include Painlevé I, II difference equations and linear difference equations. Some of these, restricting the coefficients to be constants, are studied by Chen and Shon [4], leading to the following.

Theorem A If $w$ is a transcendental finite-order meromorphic solution of

$$
\bar{w}+\underline{w}=\frac{\left(a_{1} z+a_{2}\right) w+a_{3}}{1-w^{2}}
$$

then

(อ2014 Zhang and Yi; licensee Springer. This is an Open Access article distributed under the terms of the Creative Commons Attribution License (http://creativecommons.org/licenses/by/2.0), which permits unrestricted use, distribution, and reproduction in any medium, provided the original work is properly cited. 
(i) $w$ has at most one non-zero finite Borel exceptional value for $\rho(w)>0$;

(ii) $\lambda(1 / w)=\lambda(w)=\rho(w)$.

In 2010, Ronkainen [14] gave the full classification of the family including Painlevé III difference equations in his dissertation. He showed that if the equation $\bar{w} \underline{w}=R(z, w)$ has an admissible meromorphic solution $w$ of hyper-order less than one, then either $w$ satisfies a difference Riccati equation or the equation can be transformed to four simple Painlevé III difference equations.

Most recently, the first author and Yi [15] investigated the properties of meromorphic solutions of Painlevé III difference equations given by [14]. Especially, we recall the following.

Theorem B If $w$ is a transcendental finite-order meromorphic solution of

$$
\bar{w} \underline{w}(w-1)=\eta(w-\lambda),
$$

where $\eta(\neq 0), \lambda \neq 1$ are constants, then

(i) $\lambda(w)=\rho(w)$;

(ii) if $\lambda=0$, then $w$ has at most one non-zero Borel exceptional value for $\rho(w)>0$.

Theorem C If $w$ is a transcendental finite-order meromorphic solution of

$$
\bar{w} \underline{w}(w-1)^{2}=w^{2}-\lambda w+\mu,
$$

where $\lambda \mu \neq 0$, then $\lambda(w)=\rho(w)$.

Example 1.1 The rational function $w_{1}(z)=\frac{1}{(z+1)^{2}}$ and the transcendental function $w_{2}(z)=$ $\sec ^{2} \frac{\pi z}{2}$ are solutions of difference equation

$$
\bar{w} \underline{w}(w-1)^{2}=w^{2} .
$$

0 is a Picard exceptional value of $w$, this shows that $\lambda \mu \neq 0$ is necessary in Theorem C.

The purpose of this paper is to study the Borel exceptional values of meromorphic solutions of difference equations (1.2) and (1.3). In fact, one may ask: what happens if we remove the restriction $\lambda=0$ in the second conclusion in Theorem B? The two solutions $w_{1}(z)$ and $w_{2}(z)$ of difference equation (1.3) given by Example 1.1 both have a Picard exceptional value (also a Borel exceptional value) 0 . It is natural to ask can the solutions of (1.3) have two Borel exceptional values? Corresponding to these questions, we obtain the following results as the complement of researching of Painlevé III difference equations.

Theorem 1.2 Let $w$ be a transcendental meromorphic solution of (1.2) with finite order. If $a, b$ are two Borel exceptional values of $w$ and $\lambda \neq 0$, then

(i) $a+b=1+\eta$;

(ii) $a b=\lambda$;

(iii) $\lambda=\eta^{2}$. 
Theorem 1.3 Let w be a transcendental meromorphic solution of (1.3) with finite order. If $a, b$ are two Borel exceptional values of $w$, then

(i) $a=0, b=2$;

(ii) $\bar{w}=\underline{w}$;

(iii) $w$ satisfies the difference Riccati equation $\bar{w}=\frac{w}{w-1}$.

Example 1.4 The transcendental function $w_{3}(z)=\frac{2 e^{i \pi z}}{e^{i \pi z}-1}$ is a solution of both difference equation (1.3) and the Riccati equation $\bar{w}=\frac{w}{w-1}$. Noting that $w_{3}(z)$ is a period function and has two Picard exceptional values 0 and 2 , we see that case (i) in Theorem 1.3 may occur.

\section{Some lemmas}

Halburd-Korhonen [11] and Chiang-Feng [10] investigated the value distribution theory of difference expressions. A key result, which is a difference analog of the logarithmic derivative lemma, reads as follows.

Lemma 2.1 Let $f$ be a transcendental meromorphic function of finite order and $c$ be a non-zero complex constant. Then

$$
m\left(r, \frac{f(z+c)}{f(z)}\right)+m\left(r, \frac{f(z)}{f(z+c)}\right)=S(r, f) .
$$

With the help of Lemma 2.1, the difference analogs of the Clunie and Mohon'ko lemmas are obtained.

Lemma 2.2 ([12]) Let $f$ be a transcendental meromorphic solution of finite order $\rho$ of a difference equation of the form

$$
U(z, f) P(z, f)=Q(z, f)
$$

where $U(z, f), P(z, f)$, and $Q(z, f)$ are difference polynomials such that the total degree $\operatorname{deg}_{f} U(z, f)=n \operatorname{in} f(z)$ and its shifts, and $\operatorname{deg}_{f} Q(z, f) \leq n$. If $U(z, f)$ contains just one term of maximal total degree in $f(z)$ and its shifts, then, for each $\varepsilon>0$,

$$
m(r, P(z, f))=O\left(r^{\rho-1+\varepsilon}\right)+S(r, f),
$$

possibly outside of an exceptional set of finite logarithmic measure.

Lemma 2.3 ([11,12]) Let $w$ be a transcendental meromorphic solution of finite order of the difference equation

$$
P(z, w)=0,
$$

where $P(z, w)$ is a difference polynomial in $w(z)$. If $P(z, a) \not \equiv 0$ for a meromorphic function a satisfying $T(r, a)=S(r, w)$, then

$$
m\left(r, \frac{1}{w-a}\right)=S(r, w) .
$$


We conclude this section by the following lemma.

Lemma 2.4 (See, e.g., [3, pp.79-80]) Let $f_{j}(j=1, \ldots, n)(n \geq 2)$ be meromorphic functions, $g_{j}(j=1, \ldots, n)$ be entire functions. If

(i) $\sum_{j=1}^{n} f_{j}(z) e^{g_{j}(z)} \equiv 0$;

(ii) $g_{h}(z)-g_{k}(z)$ is not a constant for $1 \leq h<k \leq n$;

(iii) $T\left(r, f_{j}\right)=S\left(r, e^{g_{h}(z)-g_{k}(z)}\right)$ for $1 \leq j \leq n$ and $1 \leq h<k \leq n$, then $f_{j}(z) \equiv 0(j=1, \ldots, n)$.

\section{Proofs of theorems}

Proof of Theorem 1.2 Rewriting (1.2) as $\bar{w} \underline{w} w=\bar{w} \underline{w}+\eta(w-\lambda)$, we get from Lemma 2.2 that $m(r, w)=S(r, w)$ and then $N(r, w)=T(r, w)+S(r, w)$. Therefore, $a$ and $b$ are not infinity.

Let $P(z, w)=\bar{w} \underline{w}(w-1)-\eta(w-\lambda)$. We get $P(z, 0)=\eta \lambda \neq 0$, Lemma 2.3 gives

$$
m(r, 1 / w)=S(r, w)
$$

which means $N(r, 1 / w)=T(r, w)+S(r, w)$ and thus $a b \neq 0$.

Now we find that $a$ and $b$ are two non-zero finite Borel exceptional values of $w$. Set

$$
f(z)=\frac{w(z)-a}{w(z)-b} .
$$

Then $\rho(f)=\rho(w), \lambda(f)=\lambda(w-a)<\rho(f)$, and $\lambda(1 / f)=\lambda(w-b)<\rho(f)$. Since $f$ is of finite order, we suppose that

$$
f(z)=g(z) e^{d z^{n}}
$$

where $d(\neq 0)$ is a constant, $n(\geq 1)$ is an integer, $g(z)$ is meromorphic and satisfies

$$
\rho(g)<\rho(f)=n .
$$

Then

$$
f(z+1)=g(z+1) g_{1}(z) e^{d z^{n}}, \quad f(z-1)=g(z-1) g_{2}(z) e^{d z^{n}},
$$

where $g_{1}(z)=e^{n d z^{n-1}+\cdots+d}$ and $g_{2}(z)=e^{-n d z^{n-1}+\cdots+(-1)^{n} d}$.

We get from (3.1) and (3.2) that $w=\frac{b f-a}{f-1}$. By (1.2) and (3.4), we have

$$
A(z) e^{3 d z^{n}}+B(z) e^{2 d z^{n}}+C(z) e^{d z^{n}}+D=0
$$

where

$$
\begin{aligned}
& A(z)=\left[b^{2}(b-1)-\eta(b-\lambda)\right] g \bar{g} g_{1} g g_{2}, \\
& B(z)=\left[b^{2}(1-a)+\eta(a-\lambda)\right] \bar{g} g_{1} g g_{2}+[a b(1-b)+\eta(b-\lambda)] g\left(\bar{g} g_{1}+g g_{2}\right), \\
& C(z)=\left[a^{2}(b-1)-\eta(b-\lambda)\right] g+[a b(a-1)+\eta(\lambda-a)]\left(\bar{g} g_{1}+g \underline{g} g_{2}\right), \\
& D=a^{2}(1-a)-\eta(a-\lambda) .
\end{aligned}
$$


From (3.3), we apply Lemma 2.4 to (3.5), and as a result all the coefficients vanish. Since $a$ and $b$ are non-zero constants, we deduce from $A(z)=0$ and $D=0$ that

$$
a^{2}(a-1)=\eta(a-\lambda), \quad b^{2}(b-1)=\eta(b-\lambda) .
$$

Then

$$
a^{3}-b^{3}=a^{2}-b^{2}+\eta(a-b) .
$$

Denote $G=g, G_{1}=\bar{g} g_{1}$, and $G_{2}=g g_{2}$. From $B(z)=0, C(z)=0$ and (3.6), we have

$$
\begin{aligned}
& (a-1)(a+b) G_{1} G_{2}=b(b-1) G\left(G_{1}+G_{2}\right), \\
& (b-1)(a+b) G=a(a-1)\left(G_{1}+G_{2}\right) .
\end{aligned}
$$

Since the last two equations are both homogeneous, there exist two non-zero constants $\alpha$ and $\beta$ such that $G_{1}=\alpha G$ and $G_{2}=\beta G$. Then

$$
\begin{aligned}
& (a-1)(a+b) \alpha \beta=b(b-1)(\alpha+\beta), \\
& (b-1)(a+b)=a(a-1)(\alpha+\beta) .
\end{aligned}
$$

On the other hand, combining (3.2) with (3.4), we get

$$
\bar{f}=\alpha f, \quad f=\beta f,
$$

which yield $\alpha \beta=1$. It follows from (3.8) and (3.9) that

$$
a(a-1)^{2}=b(b-1)^{2}
$$

Combining (3.6) and (3.10) gives

$$
(a-1) \frac{\eta(a-\lambda)}{a}=(b-1) \frac{\eta(b-\lambda)}{b} .
$$

Noting that $\eta \neq 0$ and $a \neq b$, we obtain $a b=\lambda$. This is the conclusion (ii).

Rewrite (3.10) as

$$
a^{3}-b^{3}=2 a^{2}-2 b^{2}+b-a .
$$

Combining this with (3.7), we get $a+b=1+\eta$, then (i) follows.

On the other hand, we get from (3.11) $a^{2}+a b+b^{2}=2(a+b)-1$, i.e.,

$$
(a+b-1)^{2}=a b=\lambda,
$$

which yields the conclusion (iii) by $a+b=1+\eta$.

Proof of Theorem 1.2 Denote

$$
u=\frac{1}{w}
$$


Then $u$ is a transcendental function of finite order and $T(r, u)=T(r, w)+O(1)$. Substituting $w=1 / u$ in (1.3), we obtain

$$
\bar{u} \underline{u}=(u-1)^{2}=u^{2}-2 u+1 .
$$

Both sides of (3.13) are divided by $u^{2}$, giving

$$
\frac{1}{u^{2}}=\frac{\bar{u}}{u} \frac{u}{u}+\frac{2}{u}-1
$$

It follows from Lemma 2.1 that

$$
2 m(r, 1 / u) \leq m(r, 1 / u)+S(r, u)
$$

which means that $m(r, w)=m(r, 1 / u)=S(r, w)$, and thus $N(r, w)=T(r, w)+S(r, w)$. Therefore, $a$ and $b$ are not infinity.

Let $P(z, w)=\bar{w} \underline{w}(w-1)^{2}-w^{2}$. Since $a$ and $b$ are Borel exceptional values of $w$, Lemma 2.3 tells us that

$$
\begin{aligned}
& P(z, a)=a^{2}(a-1)^{2}-a^{2}=0, \\
& P(z, b)=b^{2}(b-1)^{2}-b^{2}=0 .
\end{aligned}
$$

Solving the last two equations, it follows that $a=0$ and $b=2$. Set

$$
f(z)=\frac{w(z)-2}{w(z)} .
$$

Then $u=1 / w=(1-f) / 2$. Substituting this in (3.13), it yields

$$
\bar{f} f-f^{2}=\bar{f}+\underset{-}{f}+2 f .
$$

Since now 0 and 2 are Borel exceptional values of $w$, we still have (3.2)-(3.4). By (3.4) and the above equation, we have

$$
\left(G_{1} G_{2}-G^{2}\right) e^{2 d z^{n}}-\left(G_{1}+G_{2}+2 G\right) e^{d z^{n}}=0,
$$

where $G_{1}, G_{2}$, and $G$ are the same as before. We apply Lemma 2.4 on the last equation, resulting in all the coefficients vanish, i.e.,

$$
G_{1} G_{2}=G^{2}, \quad G_{1}+G_{2}=-2 G,
$$

from which we get $G_{1}=G_{2}=-G$. Then $\bar{f}=\underline{f}=-f$ by (3.2) and (3.4). Noting (3.14) and $w=\frac{2}{1-f}$, it follows that

$$
\bar{w}=\frac{2}{1-\bar{f}}=\frac{2}{1-f}=\underline{w}
$$


and

$$
\bar{w}=\frac{2}{1-\bar{f}}=\frac{2}{1+f}=\frac{2}{1+\frac{w-2}{w}}=\frac{w}{w-1} .
$$

\section{Competing interests}

The authors declare that they have no competing interests.

\section{Authors' contributions}

Both authors drafted the manuscript, and they read and approved the final manuscript.

\section{Author details}

'LMIB and School of Mathematics and Systems Science, Beihang University, Beijing, 100191, P.R. China. ${ }^{2}$ School of Mathematics, Shandong University, Jinan, Shandong 250100, P.R. China.

\section{Acknowledgements}

The authors would like to thank the referee for valuable suggestions to the present paper. This research was supported by the NNSF of China Nos. 11201014, 11171013, 11126036 and the YWF-14-SXXY-008, YWF-ZY-302854 of Beihang University. This research was also supported by the youth talent program of Beijing No. 29201443.

Received: 19 March 2014 Accepted: 29 April 2014 Published: 13 May 2014

\section{References}

1. Hayman, WK: Meromorphic Functions. Clarendon, Oxford (1964)

2. Laine, I: Nevanlinna Theory and Complex Differential Equations. de Gruyter, Berlin (1993)

3. Yang, CC, Yi, HX: Uniqueness Theory of Meromorphic Functions. Kluwer Academic, Dordrecht (2003)

4. Chen, ZX, Shon, KH: Value distribution of meromorphic solutions of certain difference Painlevé equations. J. Math. Anal. Appl. 364, 556-566 (2010)

5. Huang, ZB, Chen, ZX, Li, Q: On properties of meromorphic solutions for complex difference equation of Malmquist type. Acta Math. Sci., Ser. B 33(4), 1141-1152 (2013)

6. Li, S, Gao, ZS: Finite order meromorphic solutions of linear difference equations. Proc. Jpn. Acad., Ser. A, Math. Sci. 87, 73-76 (2011)

7. Li, S, Chen, BQ: Results on meromorphic solutions of linear difference equations. Adv. Differ. Equ. 2012, 203 (2012)

8. Zhang, JL, Yang, LZ: Meromorphic solutions of Painlevé III difference equations. Acta Math. Sin. Chin. Ser. 57, 181-188 (2014)

9. Zhang, JL, Yang, LZ: Entire solutions of $q$-difference equations and value distribution of $q$-difference polynomials. Ann. Pol. Math. 109, 39-46 (2013)

10. Chiang, YM, Feng, SJ: On the Nevanlinna characteristic of $f(z+\eta)$ and difference equations in the complex plane. Ramanujan J. 16, 105-129(2008)

11. Halburd, RG, Korhonen, RJ: Difference analogue of the lemma on the logarithmic derivative with applications to difference equations. J. Math. Anal. Appl. 314, 477-487 (2006)

12. Laine, I, Yang, CC: Clunie theorems for difference and q-difference polynomials. J. Lond. Math. Soc. 76, 556-566 (2007)

13. Halburd, RG, Korhonen, RJ: Finite order solutions and the discrete Painlevé equations. Proc. Lond. Math. Soc. 94, 443-474 (2007)

14. Ronkainen, O: Meromorphic Solutions of Difference Painlevé Equations. Ann. Acad. Sci. Fenn. Diss., vol. 155 (2010)

15. Zhang, JL, Yi, HX: Properties of meromorphic solutions of Painlevé III difference equations. Adv. Differ. Equ. 2013,256 (2013)

10.1186/1687-1847-2014-144

Cite this article as: Zhang and Yi: Borel exceptional values of meromorphic solutions of Painlevé III difference equations. Advances in Difference Equations 2014, 2014:144

\section{Submit your manuscript to a SpringerOpen ${ }^{\circ}$ journal and benefit from:}

- Convenient online submission

- Rigorous peer review

- Immediate publication on acceptance

- Open access: articles freely available online

- High visibility within the field

- Retaining the copyright to your article 